\title{
FORUM
}

\section{Energiepolitik für Europa - ein Schwerpunkt der deutschen Ratspräsidentschaft}

\author{
Günter Gloser*
}

Die Europäische Union befasst sich mit neuer Intensität mit der Energiepolitik. Deutschland will diese Diskussion aktiv mitgestalten. Energiepolitik wird ein Schwerpunkt der deutschen EU-Präsidentschaft im 1. Halbjahr 2007 sein. Im Vorfeld wollen wir eng mit der aktuellen finnischen Ratspräsidentschaft zusammenarbeiten.

Wichtige Impulse für die steigende Bedeutung energiepolitischer Fragestellungen gab der Europäische Rat in Hampton Court im Herbst 2005. Die zurückgehende Eigenförderung in der Nordsee begründet eine steigende Importabhängigkeit der Europäischen Union für Öl und Gas und damit eine größere Abhängigkeit von Entwicklungen außerhalb der Europäischen Union. Dies erfordert - auch durch neue starke Energienachfragezentren in Asien ein größeres Engagement der Europäischen Union und ein einheitlicheres Auftreten, nach innen wie nach außen. Gleichzeitig nehmen die Anforderungen des Klimaschutzes auch an die Energiepolitik zu. Die Europäische Union ist hier traditionell Vorreiterin unter anderem durch ambitionierte Ziele zur Reduzierung der Emissionen und durch das europäische Emissionshandelssystem, das die Emissionsminderung wirtschaftlich effizient fördern soll. Die Emissionsminderungen der Europäischen Union allein reichen allerdings nicht aus. Wir müssen das internationale Klimaschutzregime ausbauen und auf eine breitere Basis stellen, auch aus Gründen der Wettbewerbsfähigkeit. Dabei muss die Europäische Union ihre Vorreiterrolle fortführen, auch um andere Länder zu überzeugen und in ein erweitertes Klimaschutzregime einzubeziehen.

\section{Leitlinien}

Umweltverträglichkeit, Wettbewerbsfähigkeit und Versorgungssicherheit sind die drei gleichrangigen Ziele deutscher Energiepolitik. Diese Ziele sind auch Leitlinien für eine ,Energiepolitik für Europa‘. Sie gilt es unter deutscher EU-Präsidentschaft auszuarbeiten und zu konkretisieren. Energiepolitik gehört bislang nicht zu den Zuständigkeiten der Europäischen Union. Erst der Verfassungsvertrag wird eine solche Kompetenz festschreiben. Dennoch hat die EU in den vergangenen Jahren insbesondere in den Bereichen Binnenmarkt und Umwelt zahlreiche wichtige energiepolitische Beschlüsse getroffen. Markantestes Bespiel ist die Schaffung des europäischen Binnenmarkts für Strom und Gas. Er muss bis Mitte 2007 von allen Mitgliedstaaten umgesetzt werden. Der Binnenmarkt wird dann die freie Wahl von Energieversorgern auch durch private Haushalte ermöglichen. Der Stand der Liberalisierung wird auch ein Thema der deutschen Präsidentschaft sein. Die Europäische Kommission wird einen Sachstandsbericht zur Umsetzung der Binnenmarktrichtlinien vorlegen.

* Günter Gloser, Staatsminister für Europa, Auswärtiges Amt, Berlin. 


\section{Aktionsprogramm Energie}

Zentrales Element der deutschen Präsidentschaft im Energiebereich wird die Verabschiedung eines Aktionsprogramms Energie sein. Der Europäische Rat im Frühjahr 2006 hat bis zum Frühjahrs-Rat 2007 die Erarbeitung dieses umfassenden Aktionsprogramms in Auftrag gegeben und bereits einige prioritäre Aufgabenbereiche identifiziert. Die Europäische Kommission erarbeitet derzeit ein umfassendes ,Energiepaket'. Es soll Maßnahmen als Grundlage für das Aktionsprogramm vorschlagen. Wichtiger Bestandteil wird eine regelmäßige strategische Überprüfung der Energiepolitik sein. Unter dem Dach des strategischen Energieberichts will die Europäische Kommission Einzelthemen wie Marktliberalisierung, Förderung erneuerbarer Energien und saubere Kohletechnologien behandeln sowie Vorschläge für das weitere Vorgehen vorlegen.

Mögliche Kernpunkte des Aktionsprogramms schälen sich bereits heraus: die Definition neuer Ansätze einer gemeinsamen Energiepolitik und einer gemeinsamen Energiekonzeption in der Europäischen Union; der Startschuss für eine gemeinsame Diskussion über langfristige Angebots- und Nachfrageperspektiven; die Entwicklung gemeinsamer Konzepte in der Außenwirtschafts- und Außenpolitik in Energiefragen; die Verabschiedung weiterer Maßnahmen im Bereich Energieeffizienz; die Stärkung der Energieforschung; der weitere Ausbau erneuerbarer Energien. Diese Kernpunkte prägen auch die Vorbereitungen für die deutsche Präsidentschaft. Dabei besteht auch mit den EU-Partnern Einigkeit, dass die Entscheidung über den Energiemix in nationaler Verantwortung zu treffen ist.

\section{Ziele der deutschen Ratspräsidentschaft}

Weitere wichtige energiepolitische Ziele der deutschen Präsidentschaft werden sein:

- Die vollständige Umsetzung des Binnenmarkts für Strom und Gas durch alle Mitgliedstaaten. Grenzüberschreitende regionale Initiativen wie die Zusammenarbeit zwischen Deutschland, den Niederlanden, Belgien, Luxemburg und Frankreich können hier wichtige Zwischenschritte darstellen und weitere Impulse für eine engere Zusammenarbeit geben.

- Die Stärkung der Versorgungssicherheit durch interne Maßnahmen wie eine weitere Entkoppelung von Wirtschaftswachstum, Energieverbrauch und Krisenvorsorgemechanismen sowie nach außen durch einen Ausbau der internationalen Energiebeziehungen.

- Die Verbesserung der Energieeffizienz insbesondere im Gebäude- und Verkehrssektor, die in der Europäischen Union etwa 70 Prozent des Endenergieverbrauchs stellen.

- Die Fortführung des Ausbaus erneuerbarer Energien unter anderem auch für das Heizen und Kühlen sowie im Verkehrssektor durch die Überarbeitung der Biokraftstoff-Richtlinie.

- Die Energieforschung mit besonderer Aufmerksamkeit für die Entwicklung marktnaher energieeffizienter Energietechnologien wie beispielsweise moderne Kraftwerkstechnik auf der Basis von Kohle und Gas.

\section{Energieaußenbeziehungen}

Im besonderen Fokus des Auswärtigen Amts stehen naturgemäß die Energieaußenbeziehungen. Die Bedeutung der Außenpolitik wird nicht zuletzt aufgrund von Instabilitäten in vielen zentralen Energieregionen der Welt, allen voran dem Nahen und Mittleren Osten, immer augenfälliger. Dieser Bedeutungszuwachs betrifft auch die europäische Ebene. So haben der Hohe Repräsentant Javier Solana und die Europäische Kommission im Mai 2005 
das Grundlagenpapier „Eine Außenpolitik zur Förderung der EU-Interessen im Energiebereich“ vorgelegt. Diese Überlegungen werden in die EU-Präsidentschaft einfließen.

Wir streben eine stärkere Kohärenz der Energieaußenbeziehungen mit anderen Politikbereichen an, wie der Entwicklungs- und Handelspolitik sowie den Ausbau der Zusammenarbeit mit Verbraucher-, Transit- und Förderländern. Daneben soll die deutsche Präsidentschaft auch regionale Akzente setzen (unter anderem die Vertiefung der Beziehungen zu Norwegen, die Stärkung des Energiedialogs mit Nordafrika und die Weiterentwicklung der Initiativen zur Zusammenarbeit mit dem Kaspischen Raum und Zentralasien).

Russland ist und bleibt zentraler Energiepartner der Europäischen Union. Wir wollen die Energiezusammenarbeit mit Russland weiter ausbauen, auch im Rahmen der anstehenden Beratungen über ein neues Partnerschafts- und Kooperationsabkommen. Der Energie-Aktionsplan der G8 von St. Petersburg bietet hierfür einen wichtigen Bezugsrahmen. Auch mit großen Verbraucherländern wie den USA, China und Indien gilt es, die Energiezusammenarbeit zu intensivieren und durch eine Kooperation bei der Klimapolitik zu komplementieren.

Unser übergreifendes Ziel ist die Stärkung kooperativer Strukturen im Energiebereich. Sie sollen - auf funktionierenden Märkten - eine sichere, wirtschaftliche und nachhaltige Energieversorgung für Deutschland und die Europäische Union auch für die kommenden Jahrzehnte sicherstellen.

Der Bereich „Europäische Energiepolitik“ wird am Insitut für Europäische Politik im Rahmen des gemeinsam mit der ASKO EUROPA-STIFTUNG durchgeführten Projekts „Ein Europa der Bürger - Verfassung und effiziente Politik“ bearbeitet. 